\title{
Selective phosphorylation during early macrophage differentiation
}

Huoming Zhang ${ }^{1,2}$, Pei-Yuan Qian ${ }^{3}$, Timothy Ravasi ${ }^{1}$

${ }^{1}$ Division of Biological and Environmental Sciences \& Engineering, Division of Applied

Mathematics and Computer Sciences, ${ }^{2}$ Bioscience Core Laboratory, King Abdullah

University of Science and Technology, Thuwal, Kingdom of Saudi Arabia.

${ }^{3}$ School of Science, Hong Kong University of Science and Technology, Hong Kong, China

Correspondence should be addressed to: Timothy Ravasi at timothy.ravasi@kaust.edu.sa.

Keywords: Leukemia, phosphoproteome, monocyte-to-macrophage differentiation

Received: 31-Oct-2014; Revised: 08-Jul-2015; Accepted: 19-Aug-2015

This article has been accepted for publication and undergone full peer review but has not been through the copyediting, typesetting, pagination and proofreading process, which may lead to differences between this version and the Version of Record. Please cite this article as doi: 10.1002/pmic.201400511.

This article is protected by copyright. All rights reserved. 


\section{Abstract}

The differentiation of macrophages from monocytes is a tightly controlled and complex biological process. Although numerous studies have been conducted using biochemical approaches or global gene/protein profiling, the mechanisms of the early stages of differentiation remain unclear. Here we used SILAC-based quantitative proteomics approach to perform temporal phosphoproteome profiling of early macrophage differentiation. We identified a large set of phosphoproteins and grouped them as PMA-regulated and nonregulated phosphoproteins in the early stages of differentiation. Further analysis of the PMAregulated phosphoproteins revealed that transcriptional suppression, cytoskeletal reorganization and cell adhesion were among the most significantly activated pathways. Some key involved regulators of these pathways are mTOR, MYB, STAT1 and CTNNB. Moreover, we were able to classify the roles and activities of several transcriptional factors during different differentiation stages and found that E2F is likely to be an important regulator during the relatively late stages of differentiation. This study provides the first comprehensive picture of the dynamic phosphoproteome during myeloid cells differentiation, and identifies potential molecular targets in leukemic cells. 


\section{Introduction}

Monocytes originate from bone marrow and are subsequently released into the blood stream, where they play essential roles in protecting the host from invading pathogens and priming the development of the adaptive immune response. These are short-lived cells routinely undergoing spontaneous apoptosis [1]. A variety of stimuli, including macrophage colony-stimulating factor [2], retinoic acid, vitamin D [3], cytokines $[4,5]$ or pathogens $[6,7]$, can prompt monocytes to migrate into tissues and differentiate into resident macrophages, which have longer life spans and provide sustained host protection. However, the undesirable accumulation of immunosuppressive macrophages may be harmful; they can aggravate chronic inflammatory diseases, such as atherosclerosis [8], arthritis and multiple sclerosis [9], and promote tumor growth [10]. Thus, gaining a better understanding of the mechanisms underlying monocyte differentiation could provide valuable insights into tissue homeostasis and might help define new therapeutic targets for the prevention/therapy of chronic inflammatory diseases and tumor progression.

Monocytes and macrophages have distinct morphologies and cellular components [11], with latter expressing certain cell surface molecules, such as CD14 and members of CD11 families [12, 13], at higher level. Monocytes and macrophages also differ in their transcriptional programs, with differential expression seen in several pathways [14, 15]. To understand how the various signaling pathways are regulated to control the differentiation of monocytes to macrophages, some researchers have performed temporal gene expression profiling $[16,17]$. Transcription factors (TFs) and microRNAs (miRs) are generally important for maintaining specific transcriptional programs. Many TFs have been identified as being critical for the differentiation of monocytes to macrophages [11, 18-20], and a transcriptional control network of the differentiation was recently constructed [21]. Similarly, a large number of miRs have been found to mediate the differentiation of macrophages. Most of the 
identified miRs are downregulated [22-24] to promote macrophage differentiation, although the upregulation of some miRs has also been associated with this process [25].

Furthermore, the interplay between miRs and TFs has been shown to be important [26].

Compared to the above-described genomics studies, relatively few proteomic studies have been performed to assess monocyte-to-macrophage differentiation. However, proteins tend to provide a better reflection of the cellular processes that help define a given cellular phenotype. Furthermore, chemical protein modification (e.g., site-specific phosphorylation) can critically control gene activation/repression in both prokaryotes and eukaryotes [27]. In many cases, phosphorylation serves as an early signal, activating signaling pathways and directly mediating the expression/localization of TFs. The phosphorylation of TFs (e.g., NFKB and the STATs) can trigger their translocation from the cytoplasm to the nucleus, thereby, selectively activating gene expression [28]. Macrophage differentiation has been reported to correlate with the increased expression and activation of members of protein kinase C, PI3K-AKT pathway, and other intracellular signaling molecules such as MAPKs, ERK1 and ERK2 $[29,30]$. Nuclear translocation of phospholipases (PLC, -D, or -A2) or treatment with phosphatidylinositol-specific PLC is also sufficient to induce monocytic differentiation [31]. Blockage of these pathways can abrogate macrophage differentiation [31]. Despite the importance of protein phosphorylation, however, little is known about how the global phosphorylation status contributes to regulating differentiation, especially during the early stages of differentiation.

Here, we treated human leukemia THP-1 cells with phorbol-12-myristate-13-acetate (PMA) [32], to generate a well defined model of the differentiation of macrophage-like cells [33, 34]. We then monitored changes in the phosphoproteome during the early stages of differentiation (up to $1 \mathrm{~h}$ post-induction). Our results revealed several key phosphorylationmodulated regulators of the differentiation process and allowed us to identify various pathways associated with the early stages of myeloid differentiation. 


\section{Materials and Methods}

\subsection{Cell culture}

The human leukemia THP-1 cell line has properties similar to those of human peripheral blood monocytes [32] and has been widely used as a model for the study of monocyte infection [35] and monocyte-to-macrophage differentiation [36]. Here, THP-1 cell samples (ATCC, TIB-202) were prepared using commercially available kits SILAC protein identification and quantitation kit (Invitrogen, CA, USA). Briefly, the cells were cultured in SILAC RPMI 1640 base medium (Invitrogen) supplemented with "light", or isotopeincorporated "medium" or "heavy" L-Lysine and L-Arginine (Cambridge Isotope Lab, UK), $10 \%$ dialyzed fetal calf serum (Invitrogen), $2 \mathrm{mM}$ L-glutamine (Invitrogen) and $50 \mu \mathrm{M}$ of $\beta$ mercaptoethanol (Sigma, MO, USA). We then studied five time points following phorbol-12myristate-13-acetate (PMA) treatment, representing five early stages of cell differentiation: the control (without PMA; time 0) and treatment groups incubated with $100 \mathrm{nM}$ PMA for 5, 15, 30 and 60 min. Control cells were cultured in the presence of the "light" L-Lysine HCl and L-Arginine $\mathrm{HCl}$ (designated R0K0). For the 5- and 30-min PMA treatments, the cells were cultured in the presence of the "medium" L-Arginine $\mathrm{HCl}(\mathrm{U}-13 \mathrm{C} 6,98 \%)$ and L-Lysine-2HCl (4,4,5,5-D4, 96-98\%) (designated R6K4). For the 15- and 60-min PMA treatments, the cells were cultured in the presence of the "heavy" L-Arginine $\mathrm{HCl}(\mathrm{U}-13 \mathrm{C} 6,98 \%, 15 \mathrm{~N} 4,98 \%)$ and L-Lysine-2HCL (U13C6, 98\%, 15N2, 98\%) (designated R10K8). In this setting, majority of the treated cells became adherent following the treatment for 1 hour, and differentiated into macrophage-like cells after 48 hours. We performed two triplex SILAC experiments: one containing the control (ROK0), the 5-min treatment (R6K4) and the 15-min treatment (R10K8); and one containing the control, the 30-min treatment (R6K4) and the 60-min treatment (R10K8). To ensure full incorporation of the "medium" and "heavy" L-lysine and Larginine prior to PMA treatments, the cells were cultured in the relevant culture medium for 
more than 6 passages, and then seeded into $10-\mathrm{cm}$ culture dishes at a density of $1 \times 10^{6}$ cells $/ \mathrm{ml}$. After the cells were adapted to the new medium for $12 \mathrm{~h}$, they were subjected to PMA treatment (three biological replicates per condition). The cells were then harvested, pelleted, and washed three times with ice-cold PBS, and lysed with SILAC cell lysis buffer (Invitrogen). All cell lysates were collected and stored at $-80^{\circ} \mathrm{C}$ until analysis.

\subsection{Protein digestion, peptide preparation and fractionation}

Each cell lysate was thawed and centrifuged at $1000 \mathrm{~g}$ for $10 \mathrm{~min}$ at $4{ }^{\circ} \mathrm{C}$. The supernatant (containing soluble proteins) was transferred to a fresh tube and the protein content was measured using a 2D-Quant kit (GE Healthcare, UK). In one triplex SILAC experiment, we mixed approximately $1 \mathrm{mg}$ protein each from the control, the 5-min treatment and the 15-min treatment; in another, we mixed $1 \mathrm{mg}$ protein each from the control, the 30 -min treatment and the 60-min treatment. The mixtures were reduced, alkylated and subjected to in-solution trypsin digestion according to our previously published protocol (Zhang et al., 2010). The peptides were desalted using Sep-Pak C18 cartridges (Waters, MA, USA) and dried in a SpeedVac (Thermo Scientific, MA, USA).

\subsection{Phosphopeptide enrichment}

Phosphopeptides were enriched using TiO2 ProteaTip Sample Prep Kit (Proteabio, Morgantown, WV, USA) according to the product manual. Briefly, the protein digests were reconstituted into $200 \mu \mathrm{L}$ of sample reconstitution and wash 1 solution. They were loaded into pre-washed TiO2 tips and centrifuged at $4000 \mathrm{~g}$ for $3 \mathrm{~min}$. The tips were washed twice with sample reconstitution and wash 1 solution to elute salts and other loosely bound components. Subsequently, they were washed twice with the wash 2 solution. The tips were then transferred to new centrifuge tubes for phosphopeptide elution by adding $200 \mu \mathrm{L}$ of elution solution. The eluted peptides were dried in a SpeedVac prior to fractionation by 
ERLIC chromatography.

In the ERLIC fractionation, the dried peptides were reconstituted in ERLIC buffer A (10 mM sodium methylphosphonate in $70 \% \mathrm{ACN}, \mathrm{pH} 2.0$ ). The separation was performed using a PolyLC PolyWAX LP ${ }^{\mathrm{TM}}$ column $(4.6 \times 200 \mathrm{~mm}, 5 \mu \mathrm{m}$ particle sizes, $300 \AA$ pore size $)$ on an Accela HPLC system (Thermo Scientific). The gradient was created using a combination of ERLIC buffer $A$ and buffer $B(200 \mathrm{mM}$ triethylamine phosphate with $60 \% \mathrm{ACN}, \mathrm{pH} 2.0)$. The 45-min gradient was composed of 5 min of $100 \%$ buffer A, 30 min ramping to $100 \%$ B and $10 \mathrm{~min}$ of $100 \% \mathrm{~B}$. The eluted fractions were monitored through a UV detector at $214 \mathrm{~nm}$ wavelength and fractions were collected at 1-min intervals. They were desalted and dried prior MS analysis.

\subsection{Mass spectrometry analysis}

The peptides were reconstituted in $10 \mu \mathrm{L}$ of $0.1 \%$ formic acid in $\mathrm{H}_{2} \mathrm{O}$ and analyzed three times using an LTQ-Orbitrap Velos (Thermo Scientific) coupled with a Proxeon EASY-nLC (Thermo Scientific). For each analysis, $3 \mu \mathrm{L}$ of the sample was injected and concentrated in a preconditioned column $(0.3 \times 50 \mathrm{~mm})$ packed with $\mathrm{C} 18 \mathrm{AQ}(5 \mu \mathrm{m}$ particles, $200 \AA$ pore size, Bruker-Michrom, CA, USA). Peptide separation was performed in a preconditioned capillary column $(0.1 \times 150 \mathrm{~mm}$, containing $\mathrm{C} 18 \mathrm{AQ}, 3-\mu \mathrm{m}$ particles, $200-\AA ̊ \AA$ pore size; Bruker-Michrom). Mobile phase A ( $0.1 \%$ formic acid in $\left.\mathrm{H}_{2} \mathrm{O}\right)$ and mobile phase $\mathrm{B}(0.1 \%$ formic acid in acetonitrile) were used to establish a 75-min gradient as follows: 45 min of 0$35 \% \mathrm{~B} ; 15 \mathrm{~min}$ of $35-80 \% \mathrm{~B}$; and $15 \mathrm{~min}$ of $80 \% \mathrm{~B}$. The total flow rate of the gradient was set at $500 \mathrm{~nL} / \mathrm{min}$. The sample was introduced into the LTQ-Orbitrap through an ADVANCE CaptiveSpray Source (Bruker-Michrom) with an electrospray potential of $1.5 \mathrm{kV}$. The ion transfer tube temperature was set at $160^{\circ} \mathrm{C}$. The LTQ-Orbitrap was directed to perform datadependent acquisition in the positive ion mode. A full MS scan with a mass range of 350$1600 \mathrm{~m} / \mathrm{z}$ was acquired in the Orbitrap at a resolution of 60,000 (at $400 \mathrm{~m} / \mathrm{z}$ ) using the profile 
mode, a maximum ion accumulation time of $1 \mathrm{sec}$ and a target value of $1 \times$ e6. Charge state screening for precursor ions was activated. The 10 most intense ions that were above a 1000-count threshold and carried multiple charges were selected for fragmentation (MS/MS) via either with collision-induced dissociation (CID) in the linear ion trap or higher energy collision dissociation (HCD) in the Orbitrap. Dynamic exclusion for both CID and HCD fragmentation was activated with a repeat count of two, an exclusion duration of $45 \mathrm{~s}$, and a mass tolerance of $\pm 5 \mathrm{ppm}$. For CID, the settings included a maximum ion accumulation time of $200 \mathrm{~ms}$ for MS/MS spectrum collection, a target value of $1 \times \mathrm{e} 4$, a normalized collision energy at $35 \%$, an activation $Q$ of 0.25 , an isolation width of 3.0 , and an activation time of 10 ms. For HCD, the settings included a maximum ion accumulation time of $200 \mathrm{~ms}$ for MS/MS spectrum collection, a target value of $5 \times$ e4, a normalized collision energy of $40 \%$, an isolation width of 3.0 , and an activation time of $0.1 \mathrm{~ms}$.

\subsection{Mass spectrometry data analysis}

The raw MS data from the LTQ-Orbitrap were processed using Maxquant (version 1.2.0.18) [37], which incorporates the Andromeda peptide search engine [38]. The IPI human database (version 3.82, 92104 sequences) and 262 common contaminant sequences was combined and used for the database search. Carbamidomethylation at cysteine residues was set as a fixed modification. The variable modifications included oxidation at methionine residues; phosphorylation at serine, threonine or tyrosine residues; and $\mathrm{N}$-terminal protein acetylation. The multiplicity was set to three and the maximum number of labeled amino acids was three per peptide. The enzyme limits were set at full trypsin cleavage. A maximum of two missed cleavages was allowed. A positive peptide was required to contain a minimum of six amino acids and a maximum of five modifications. The mass tolerances of the precursor ion were set to $20 \mathrm{ppm}$ and $6 \mathrm{ppm}$ for the first and main searches respectively. The top 10 parent ion pairs per $100 \mathrm{Da}$ were set for quantification. The mass tolerances of the fragments were $20 \mathrm{ppm}$ for HCD and 0.5 Da for CID. The false discovery rates (FDRs) of 
the peptides, sites and proteins were all set to 0.01. For protein quantification, we used peptides including unmodified peptides, phosphorylated peptides, oxidized methionines and $\mathrm{N}$-terminal protein acetylation. The minimum peptides and ratio counts were set to two, and proteins were required to contain at least one razor peptide. The least modified peptides and normalized ratios were used for site quantification. The re-quantify option in MaxQuant was selected. The five-time-point profile was constructed from the two sets of three time-point experiments, in which experiment 1 included the control, 5-min and 15-min, and experiment 2 included the control, 30-min and 60-min groups. Profiles were constructed using Perseus (version 1.2.0.17 from the http://www.maxquant.org/).

\subsection{SWATH-MS analysis}

The cell preparation and PMA-treatment were same as in the SILAC experiments except that all cells were cultured in the presence of the "light" L-Lysine $\mathrm{HCl}$ and L-Arginine $\mathrm{HCl}$. Phosphopeptides were prepared as above-described for SILAC experiment but without ERLIC fractionation.

The peptides were analyzed once with an IDA-mode acquisition and three times in SWATHmode acquisition using a Triple TOF 5600 Plus System (AB SCIEX, USA) coupled with an UltiMate $^{\text {TM }} 3000$ UHPLC (Thermo Scientific). For each analysis, the peptide sample was injected and concentrated in a Nano-trap column (PepMap100, $100 \mu \mathrm{m}$ i.d., $5 \mu \mathrm{m}$ of C18 particle size and $300 \AA$ A pore size, Thermo Scientific). The peptide separation was performed in a C18 column (Acclaim PepMap100, 75 m I.D. X 15 cm, $3 \mu \mathrm{m}$ particle sizes, $100 \AA ̊$ pore sizes). Mobile phase $A\left(0.1 \%\right.$ formic acid in $\left.\mathrm{H}_{2} \mathrm{O}\right)$ and mobile phase $\mathrm{B}(0.1 \%$ formic acid in $80 \% \mathrm{ACN}$ ) were used to establish a 75 -min gradient comprised of 55 min of $5-45 \% \mathrm{~B}, 4-\mathrm{min}$ ramping to $98 \% \mathrm{~B}, 6 \mathrm{~min}$ of $98 \% \mathrm{~B}$ and $10 \mathrm{~min}$ of $2 \% \mathrm{~B}$ for column conditioning. The column temperature is $40^{\circ} \mathrm{C}$ constantly and the total flow rate of the gradient was $300 \mathrm{~nL} / \mathrm{min}$. The sample was introduced into MS through a Nanospray III source (AB Sciex, USA) with an 
electrospray potential of $2.4 \mathrm{kV}$. The ion source setting includes an interface heater temperature of $150^{\circ} \mathrm{C}$, a curtain gas of $25 \mathrm{PSI}$, and a nebulizer gas of $6 \mathrm{PSI}$. In the IDAmode, the mass range of survey scans was set to 395-1250 Da. The top 30 ions of high intensity with over a threshold of 500 counts per second (counts/s) and a $2^{+}$to $4^{+}$chargestate were selected for MS/MS. A rolling collision energy option was applied. The maximum cycle time was fixed to $2 \mathrm{~s}$. A maximum accumulation time was $250 \mathrm{~ms}$ for parent ions and $150 \mathrm{~ms}$ for product ions. Dynamic exclusion was set as $15 \mathrm{~s}$ with a $50 \mathrm{mDa}$ of mass tolerance. In the SWATH-acquisition, a total of 32 SWATH windows at width of 25.0 Da were used within TOF mass range from 400.0 Da to $1200.0 \mathrm{Da}$, and accumulation time was 250 $\mathrm{ms}$ for TOF MS and $100 \mathrm{~ms}$ for product ions. This resulted a total duty cycle time of $3.5 \mathrm{~s}$.

The IDA MS data were converted into mascot generic files using Protein Pilot Software v4.5 (AB Sciex) before the database search using the Mascot (version 2.3, Matrix Sciences Ltd., London, UK) against the IPI human database. The enzyme limits were set at full tryptic cleavage at both ends, and a maximum of one missed cleavage was allowed. The mass tolerances were set $50 \mathrm{ppm}$ for the precursor ions and 0.2 Da for the fragment ions. Fixed modification was iodoacetylamide at cysteine, whereas variable modifications for the search include phosphorylation at serine, threonine or tyrosine, and oxidation at methionine residue. The search results were processed by the peptide prophet algorithm [39] built in transproteomics pipeline (TPP) for validation of peptide identifications. A spectral library for the identified peptides was built using Skyline (v3.1). All SWATH data were then analyzed using Skyline against the spectral library for peptide identification and quantitation as illustrated in Figure S1. 


\subsection{Bioinformatic analyses}

The differentially expressed phosphoproteins and total identified phosphoproteins were submitted to the web-based platform of the Database for Annotation, Visualization and Integrated Discovery (DAVID; http://david.abcc.ncifcrf.gov/) for Gene Ontology (GO) enrichment analysis and prediction of TF binding sites. The heatmap of differentially expressed phosphoproteins was constructed using the MultiExperimentView software (version 4.7.3) [40]. Protein-protein interactions were predicted using the STRING database (http://string-db.org/, version 9.0).

\section{Results and discussion}

\subsection{Phosphoprotein identification and quantification}

To uncover the mechanisms governing monocyte-to-macrophage differentiation, we measured the global protein phosphorylation changes during early differentiation. As phosphopeptides are normally found at a relatively low abundance in a typical complex protein digest, we enriched for phosphopeptides using a combination of $\mathrm{TiO}_{2}$ and $\mathrm{ERLIC}$ (electrostatic repulsion-hydrophilic interaction chromatography). For phosphopeptide quantification, we adopted a SILAC (stable isotope labeling by amino acids in cell culture) strategy, which is one of most widely used quantitative proteomic approaches (Figure 1A). With this strategy, we identified a total of 1173 phosphorylation sites on 691 phosphoproteins (Table S1). Among them, 876 sites had localization scores $>0.75$ (with a median of 0.9998); these were classified as group I sites, as described by Olsen et al. [41] (Figure 1B). Most of the identified phosphopeptides had a single phosphorylation site, but we also identified some peptides with $>3$ sites (Figure 1C). The identified phosphorylation sites consisted of $88.1 \%$ serine, $9.2 \%$ threonine, and $2.6 \%$ tyrosine (Figure 1D). This distribution 
is similar to the theoretical estimation [42] and results from other high-throughput phosphoproteomic studies [41, 43,44], indicating that we obtained a non-biased recovery of the phosphoproteome.

Of the proteins containing group I sites $(n=639), 149$ proteins showed site perturbation with a $>$ 2-fold change for at least at one time point; these were considered to be PMA-regulated phosphoproteins (Table S2). The remaining 490 phosphoproteins were defined as nonregulated phosphoproteins, at least during the early stages of PMA-induced monocyte-tomacrophage differentiation. Using SWATH-MS approach, we detected 32 phosphopeptides that overlap with those PMA-regulated phosphopeptides. Among them, 24 showed similar perturbation pattern (Figure 2); 5 phosphopeptides have inaccurate SWATH quantitation due to large variations in technical replicates and three peptides (MCM2: GNDPLTSS(ph)PGR; CCTB: MLQALS(ph)PK; EIF4EBP1: NS(ph)PVTKT(ph)PPR) showed conflict results to the SILAC data.

\subsection{Functional analysis of the phosphoproteome}

To gain additional insights into the phosphorylation-related regulation that occurred during the early differentiation of monocytes to macrophages, we used DAVID functional annotation tools to perform GO enrichment analysis of the PMA-regulated and non-regulated phosphoproteins. The top 20 enriched GO terms were plotted versus their enrichment $p$ values for the terms cellular components, molecular functions and biological pathways. The pattern was similar between the regulated and non-regulated groups for the cellular component category (Figure 3A). In both groups, the enriched GO terms were related to a variety of cellular components, including the cytosol, organelles, nucleus, chromatin, cytoskeleton and membrane. The most significant enrichments included intracellular membrane-bound organelles, non-membrane-bound organelles, cytosol and nucleus. Interestingly, the extrinsic-to-membrane category was significantly enriched in the regulated 
phosphoprotein group but not in the non-regulated phosphoprotein group, suggesting that the phosphorylation of membrane receptors is an important step in initiating the differentiation process in this system. Distinct patterns were observed for the molecular function and biological pathway groups. As shown in Figure 3B, enzyme activators and GTPase regulators were the most significantly enriched categories among the regulated phosphoproteins, whereas RNA binding, nucleotide and nucleoside binding were the top enriched GO terms in the non-regulated group. Rho/Ras guanyl-nucleotide exchange factor activity, which stimulates the exchange of guanyl nucleotides associated with a GTPase, was enriched only in the regulated group. With respect to biological pathway enrichment, the most significant terms for the regulated phosphoproteins were those involved in signal transduction (e.g., intracellular signaling cascades, regulation of Ras proteins and smallGTPase-mediated signal transduction), cytoskeletal organization (e.g., actin cytoskeleton and actin-filament-based processes) and the cell cycle (e.g., cell cycle processes, mitotic cell cycle, chromosomal organization and cell death). The non-regulated group, in contrast, was enriched in categories related to RNA metabolisms (e.g., RNA/mRNA splicing, processing, transport, and localization) (Figure 3C).

\subsection{Phosphorylation-controlled transcriptional regulation}

Cell differentiation requires the transcriptional activation of numerous genes, and the binding of TFs to specific regulatory sequences in the genome is essential for gene activation. Protein phosphorylation often alters a protein's conformation, subcellular localization and/or (in case of TFs) DNA-binding affinity. To identify the key TFs that could be involved in medicating the monocyte-to-macrophage differentiation process, we used a weighted matrixes approach to scan the promoter sequences of the regulated and non-regulated phosphoprotein groups for enrichment of transcription factor binding sites (TFBSs). Significant enrichments, which were obtained using the UCSC_TFBS option in DAVID (with $p$-value $<0.01$ ), are listed in Supplemental Tables 3 and 4. Although there were difference in 
fold enrichments and significances of the predicted TFBSs, we observed generally similar pattern of TFBSs for both protein groups, indicating that phosphoproteins may play a general regulated role during macrophage differentiation. Many of the TFs identified herein as putative regulators had been previously associated with this differentiation process $[11,20]$. In particular, 22 of the identified TFs are included in a 30-TF core transcriptional network that was previously identified using microarray and RNA sequencing approaches [21]. Moreover, the significances of the TFBSs enriched in our non-regulated phosphoprotein group were very similar to those previously found when changes in gene expression were monitored from $1 \mathrm{~h}$ to $48 \mathrm{~h}$ after the induction of differentiation, with E2F being the most significantly enriched TF [21]. Although we did not identify any specific regulatory role for this group of proteins during the early differentiation period, our results suggest that they may play a more central role in transcriptional control during the later stages of differentiation process. In the PMA-regulated protein group, STAT1 and MYB were the two top enriched TFs with nearly $10 \%$ higher enrichment compared to their level in the non-regulated phosphoprotein group. Interestingly GO analysis of the STAT1-regulated proteins showed enrichment of regulation of small GTPase signal transduction, protein kinase cascades, regulation of apoptosis and cell death, whereas that for the MYB-regulated proteins showed enrichment for cytoskeletal modeling and small GTPase activity.

STAT1 and MYB are known to play essential roles in cell differentiation [46, 47], and increased phosphorylation of STAT1 is known to be required for monocyte to macrophage differentiation [48]. Monocyte adhesion during an early stage of differentiation activates STAT1, which subsequently controls the expression level of other genes that are responsible for controlling cell adhesion (e.g., ICAM-1 and FcyRI) [49]. The nuclear translocation of STAT1 via an interaction with nucleolin was shown to be essential during the late stages of monocyte differentiation, but STAT1 phosphorylation was not required for this process [50]. The MYB family of TFs consists of three members (c-, a- and v-MYB). Downregulation of cMYB is required for the differentiation of myeloid cells and erythroid leukemia cells [51-53], 
whereas constitutive expression of c-MYB can block the differentiations of these cells [5456]. In our SWATH-MS data, we found increased phosphorylation at the Ser727 residue of STAT1 and decreased phosphorylation at the Ser1163 residue of MYBBP1A (Figure 4). Thus previous studies and our findings indicate that STAT1 and MYB family members may play central roles in regulating monocyte adhesion and cell morphology remodeling during the early stages of the differentiation. This is also consistent with the rapid cell adhesion and morphological changes observed upon the induction of differentiation [57]. Based on the available evidences, we propose that modulating the expression or phosphorylation of STAT1 and MYB family members could represent an effective means for controlling monocyte differentiation and reduce the accumulation of macrophages in inflammatory diseases.

\subsection{The protein network responsible for controlling macrophage differentiation}

Many proteins work in complexes with other proteins. Thus, studying protein-protein interactions can better explain the role of a protein in a biological context such as cell differentiation. Here, we used the STRING database to probe the protein-interaction capabilities of the 149 identified regulated phosphoproteins. Our results allowed us to infer an interacting network composing 101 nodes and 175 edges. This network could be further partitioned into five distinct sub-networks by K-mean clustering of the nodes based on their expression profiles across differentiation (Figure 5). A central hub protein could be identified for each of the five sub-networks: mTOR, PRKACA, MAPK14, CTNNB1 and ARHGEF7 formed the hubs for the sub-networks A, B, C, D and E respectively (Figure 4). 
Sub-network A contained proteins that are mainly involved in transcription and translation. MTOR (mammalian target of rapamycin) is a serine-threonine kinase that plays a pivotal role in regulating protein translation, the actin cytoskeleton, cell survival and cell growth [58], and controls the synthesis of the translational machinery by phosphorylating the ribosomal p70S6 kinase and EIF4E-BP [59]. Blockage of mTOR signaling by histone deacetylase inhibitor treatment was reported to trigger noticeable HL-60 cell differentiation [60], while overexpression of RTP801, a negative regulator of $\mathrm{mTOR}$, is required for the retinoic acidinduced differentiation in U937 cells [61].

Sub-network B comprises a large group of proteins: CTNNB1 is the central hub, suggesting that it may play a crucial role in monocyte-to-macrophage differentiation. A previous study showed that stabilization or accumulation of CTNNB1 was sufficient to specify the differentiation of FDB1 cells to macrophages [62], and CTNNB1 is known to regulate transcription via the canonical Wnt/ $\beta$-catenin pathway [63]. A number of proteins involved in this pathway have been identified, including TRRAP, which responsible for the transcriptional activation of c-MYC; MEN1, which is involved in DNA repair; and the scaffold proteins, SLC9A3R1, IQGAP1 and LIMA1, which regulate the actin cytoskeleton and may enhance Wnt signaling [64]. CTNNB1 can also form a cadherin/ $\beta$-catenin complex that can be used to generate adherent junctions, and thus act as a key player connecting cellular adhesion to signal transduction pathways [65]. In the present study, we identified a number of regulatory phosphoproteins that had functions associated with cell adhesion. For example, CD44 (a $\beta$-catenin-associated partner) is dephosphorylated at the Ser325 residues. Notably this dephosphorylation is known to increase the binding of ERM proteins to CD44 [66]. TJP2 and ZYX are important components for tight junctions and focal adhesions respectively [67, 68]. 
Sub-network C includes five proteins (ARHGEF1, -6, and -7, GIT1 and NCK1) that are likely to be involved in cell migration, attachment, adhesion and cytoskeletal reorganization. ARHGEF1, -6 and -7 belong to the Rho GTPase family, whose member act via G protein coupled receptors to play fundamental roles in numerous cellular processes initiated by extracellular stimuli. They form complexes with $\mathrm{G}$ proteins and stimulate Rho-dependent signals. ARHGEF1 contributes to the regulation of RhoA GTPase [69] and ARHGEF6 and -7 form a complex with the small GTP binding protein, RAC1, and recruiting it to membrane ruffles and focal adhesion sites. ARHGEF6 determines the podosome size and number in macrophages [70]. Finally, GIT1 and NCK1 can serve as scaffolds for signaling complexes that control vesicle trafficking, cell adhesion and cytoskeletal organization, thereby increasing the speed of cell migration [71, 72].

Sub-networks $D$ and $E$ both have protein kinases as their central nodes. PRKACA, the hub of sub-network $\mathrm{D}$, confers a broad range of functions by phosphorylating numerous substrates in the cytoplasm and nucleus. It also appears to play roles in the regulation of lipid metabolism and adipocyte differentiation. For example, PRKACA forms a complex with PPARy, thereby acting as an important regulatory protein for an efficient lipolytic response [73]. Dysregulation of PRKACA results in metabolic perturbation in mutant mice and obese peoples [73]. Activation of PRKACA increases adipogenic differentiation and inhibits osteogenic differentiation under a high-glucose regime [74], and can directly promote the adipocyte differentiation of human mesenchymal stem cells [75]. Thus, we speculate that PRKACA may play a specific role in regulating lipid metabolism, which is an important component of macrophage differentiation [76]. The p38 (MAKP14; the hub of sub-network E) also plays a central role by phosphorylating ERK2/ERK1, downstream substrates of $\mathrm{p38}$, in a time-dependent manner during the monocyte-to-macrophage differentiation. In addition, p38 MAPK signaling regulates MEF2D during differentiation and cell growth, cell survival and apoptosis $[18,77,78]$. 


\section{Conclusions}

Monocyte-to-macrophage differentiation is an intriguing but complex biological process that is coordinately regulated by transcriptional and translational events, post-translational protein modification and protein-protein interactions. Gaining a better understanding of this process will helpfully provide new insights into the developmental differentiation of myeloid cell and tissue homeostasis, and can lead to the identification of new targets in the battles against chronic inflammatory diseases and tumor progression. In this regard, numerous studies have been conducted using either conventional biochemical approaches or genome-wide expression profiling of genes and proteins. To the best of our knowledge, however, this study represents the first temporal phosphoproteome analysis of early phases of the monocyte-to-macrophage differentiation. We used SILAC-based proteomics to identify a large set of phosphoproteins, and then classified them as PMA-regulated or non-regulated proteins during the early phases of differentiation. Many of regulated phosphosites were further verified by the SWATH-MS approach. Several of the identified proteins were members of the protein kinase $\mathrm{C}$ family, which were previously shown to play a central role in monocyte-to-macrophage differentiation [79]. This indirectly supports the effectiveness and validity of our approach.

Monocytes differ from macrophages in their morphologies and cellular components. The monocyte-to-macrophage transition is thus accompanied by the synthesis of new molecules and reorganization of the cellular machinery. Interestingly, although active transcription of membrane receptors, signal transducers and extracellular proteins has been observed at late differentiation phases [16], we found several key transcription factors that must be phosphorylated in order to control transcription were dephosphorylated during the early phase of differentiation. The inferred protein-protein interaction network for the phosphorylated proteins also revealed a general downregulation of transcription, with mTOR 
as a central regulator. Moreover, proteins involved in RNA processing were enriched only in the non-regulated phosphoprotein group, indicating that mRNA translation was inactivated. In contrast, many proteins involved in cell morphology and cytoskeletal organization seemed to be mainly controlled by phosphorylation in our studied system. Our GO and PPI analyses both identified cytoskeletal-organization related proteins as being significantly represented in the PMA-regulated group (Fig. 3C and 5). For example CTNNB was identified as a central regulator of the network. These findings were further supported by our TFBS analysis. A previous report had identified a regulatory network composed of 30 core TFs that controls monocyte-to macrophage differentiation [21]. In the present study, we recovered most of these TFs from both the PMA-regulated and non-regulated phosphoprotein groups. Notably, certain TFs (such as member of the E2F family) were highly enriched in the non-regulatory phosphoprotein group, whereas TFs contribute to controlling cytoskeletal organization and cell adhesion (e.g., STAT1 and MYB) were highly enriched in the PMA-regulated phosphoproteins.

In summary, we herein showed that transcriptional suppression and cytoskeletal reorganization are the two most significant events associated with the early stages of monocyte-to-macrophage differentiation. We also classified several TFs based on their roles in different stages of differentiation.

\section{Acknowledgments}

We thank Charalampos Harris Mavromatis for his help for improving the Figure 5 . This work was supported by a grant from The King Abdullah University of Science and Technology, Thuwal, Kingdom of Saudi Arabia, to Timothy Ravasi. 


\section{References}

[1] Parihar, A., Eubank, T. D., Doseff, A. I., Monocytes and macrophages regulate immunity through dynamic networks of survival and cell death. J.Innate Immun. 2010, 2, 204-215. [2] Metcalf, D., Burgess, A. W., Clonal analysis of progenitor cell commitment of granulocyte or macrophage production. J. Cell. Physiol. 1982, 111, 275-283.

[3] Friedman, A. D., Transcriptional control of granulocyte and monocyte development. Oncogene 2007, 26, 6816-6828.

[4] Krutzik, S. R., Hewison, M., Liu, P. T., Robles, J. A., et al., IL-15 links TLR2/1-induced macrophage differentiation to the vitamin D-dependent antimicrobial pathway. J. Immunol. 2008, 181, 7115-7120.

[5] Netea, M. G., Lewis, E. C., Azam, T., Joosten, L. A., et al., Interleukin-32 induces the differentiation of monocytes into macrophage-like cells. Proc. Natl. Acad. Sci. U S A 2008, $105,3515-3520$.

[6] Adams, D. O., The structure of mononuclear phagocytes differentiating in vivo. II. The effect of Mycobacterium tuberculosis. Am. J. Pathol. 1975, 80, 101-116.

[7] Petit, A. J., Terpstra, F. G., Miedema, F., Human immunodeficiency virus infection downregulates HLA class II expression and induces differentiation in promonocytic U937 cells. J. Clin. Invest. 1987, 79, 1883-1889.

[8] Williams, H. J., Fisher, E. A., Greaves, D. R., Macrophage differentiation and function in atherosclerosis: opportunities for therapeutic intervention? J.Innate Immun. 2012, 4, 498508.

[9] Linker, R., Gold, R., Luhder, F., Function of neurotrophic factors beyond the nervous system: inflammation and autoimmune demyelination. Crit. Rev.Immunol. 2009, 29, 43-68. [10] Lamagna, C., Aurrand-Lions, M., Imhof, B. A., Dual role of macrophages in tumor growth and angiogenesis. J. Leukoc Biol. 2006, 80, 705-713.

[11] Valledor, A. F., Borras, F. E., Cullell-Young, M., Celada, A., Transcription factors that regulate monocyte/macrophage differentiation. J. Leukoc Biol. 1998, 63, 405-417. 
[12] Schwende, H., Fitzke, E., Ambs, P., Dieter, P., Differences in the state of differentiation of THP-1 cells induced by phorbol ester and 1,25-dihydroxyvitamin D3. J. Leukoc. Biol. $1996,59,555-561$.

[13] Kim, J., Feldman, R. A., Activated Fes protein tyrosine kinase induces terminal macrophage differentiation of myeloid progenitors (U937 cells) and activation of the transcription factor PU.1. Mol. Cell. Biol. 2002, 22, 1903-1918.

[14] Dong, C., Zhao, G., Zhong, M., Yue, Y., et al., RNA sequencing and transcriptomal analysis of human monocyte to macrophage differentiation. Gene 2013, 519, 279-287.

[15] Shimizu, T., Kuromi, A., Takeda, K., Synergistic induction of gene expression during the differentiation into mature macrophage in human myeloblastic leukemia cells treated with TPA and KH1060. Leuk. Res. 2009, 33, 803-809.

[16] Martinez, F. O., Gordon, S., Locati, M., Mantovani, A., Transcriptional profiling of the human monocyte-to-macrophage differentiation and polarization: new molecules and patterns of gene expression. J. Immunol. 2006, 177, 7303-7311.

[17] Lehtonen, A., Ahlfors, H., Veckman, V., Miettinen, M., et al., Gene expression profiling during differentiation of human monocytes to macrophages or dendritic cells. J. Leukoc. Biol. 2007, 82, 710-720.

[18] Baek, Y. S., Haas, S., Hackstein, H., Bein, G., et al., Identification of novel transcriptional regulators involved in macrophage differentiation and activation in U937 cells. BMC Immunol. 2009, 10, 18.

[19] Aude-Garcia, C., Collin-Faure, V., Bausinger, H., Hanau, D., et al., Dual roles for MEF2A and MEF2D during human macrophage terminal differentiation and c-Jun expression. Biochem. J. 2010, 430, 237-244.

[20] Huber, R., Pietsch, D., Gunther, J., Welz, B., et al., Regulation of monocyte differentiation by specific signaling modules and associated transcription factor networks. Cell. Mol Life Sci. : CMLS 2013. 
[21] Consortium, F., Suzuki, H., Forrest, A. R., van Nimwegen, E., et al., The transcriptional network that controls growth arrest and differentiation in a human myeloid leukemia cell line. Nat. Genet. 2009, 41, 553-562.

[22] Sonda, N., Simonato, F., Peranzoni, E., Cali, B., et al., miR-142-3p prevents macrophage differentiation during cancer-induced myelopoiesis. Immunity 2013, 38, 1236 1249.

[23] Pospisil, V., Vargova, K., Kokavec, J., Rybarova, J., et al., Epigenetic silencing of the oncogenic miR-17-92 cluster during PU.1-directed macrophage differentiation. EMBO J. $2011,30,4450-4464$.

[24] Li, T., Morgan, M. J., Choksi, S., Zhang, Y., et al., MicroRNAs modulate the noncanonical transcription factor NF-kappaB pathway by regulating expression of the kinase IKKalpha during macrophage differentiation. Nat. Immunol. 2010, 11, 799-805. [25] Munoz-Pacheco, P., Ortega-Hernandez, A., Miana, M., Cachofeiro, V., et al., Ezetimibe inhibits PMA-induced monocyte/macrophage differentiation by altering microRNA expression: a novel anti-atherosclerotic mechanism. Pharmacol. Res.2012, 66, 536-543. [26] Schmeier, S., MacPherson, C. R., Essack, M., Kaur, M., et al., Deciphering the transcriptional circuitry of microRNA genes expressed during human monocytic differentiation. BMC genomics 2009, 10, 595.

[27] Karin, M., Hunter, T., Transcriptional control by protein phosphorylation: signal transmission from the cell surface to the nucleus. Curr. Biol. 1995, 5, 747-757.

[28] Perkins, N. D., Post-translational modifications regulating the activity and function of the nuclear factor kappa B pathway. Oncogene 2006, 25, 6717-6730.

[29] Grasset, M. F., Gobert-Gosse, S., Mouchiroud, G., Bourette, R. P., Macrophage differentiation of myeloid progenitor cells in response to M-CSF is regulated by the dualspecificity phosphatase DUSP5. J. Leukoc. Biol. 2010, 87, 127-135.

[30] Stixova, L., Prochazkova, J., Soucek, K., Hofmanova, J., Kozubik, A., 5-Lipoxygenase inhibitors potentiate 1alpha,25-dihydroxyvitamin D3-induced monocytic differentiation by activating p38 MAPK pathway. Mol. Cell. Biochem. 2009, 330, 229-238. 
[31] Hughes, P. J., Marcinkowska, E., Gocek, E., Studzinski, G. P., Brown, G., Vitamin D3driven signals for myeloid cell differentiation--implications for differentiation therapy. Leuk. Res. 2010, 34, 553-565.

[32] Heil, T., Volkmann, K., Wataha, J., Lockwood, P., Human peripheral blood monocytes versus THP 1 monocytes for in vitro biocompatibility testing of dental material components. J. Oral. Rehabil. 2002, 29, 401-407.

[33] Kodama, T., Freeman, M., Rohrer, L., Zabrecky, J., et al., Type I macrophage scavenger receptor contains alpha-helical and collagen-like coiled coils. Nature 1990, 343, 531-535.

[34] Rohrer, L., Freeman, M., Kodama, T., Penman, M., Krieger, M., Coiled-coil fibrous domains mediate ligand binding by macrophage scavenger receptor type II. Nature 1990, $343,570-572$

[35] Fontan, P., Aris, V., Ghanny, S., Soteropoulos, P., Smith, I., Global transcriptional profile of Mycobacterium tuberculosis during THP-1 human macrophage infection. Infect. Immun. $2008,76,717-725$.

[36] Auwerx, J., The human leukemia cell line, THP-1: a multifacetted model for the study of monocyte-macrophage differentiation. Experientia 1991, 47, 22-31.

[37] Cox, J., Mann, M., MaxQuant enables high peptide identification rates, individualized p.p.b.-range mass accuracies and proteome-wide protein quantification. Nat. Biotechnol. 2008, 26, 1367-1372.

[38] Cox, J., Neuhauser, N., Michalski, A., Scheltema, R. A., et al., Andromeda: a peptide search engine integrated into the MaxQuant environment. J. Proteome Res. 2011, 10, 17941805.

[39] Keller, A., Nesvizhskii, A. I., Kolker, E., Aebersold, R., Empirical statistical model to estimate the accuracy of peptide identifications made by MS/MS and database search. Anal. Chem. 2002, 74, 5383-5392.

[40] Saeed, A. I., Sharov, V., White, J., Li, J., et al., TM4: a free, open-source system for microarray data management and analysis. BioTechniques 2003, 34, 374-378. 
[41] Olsen, J. V., Blagoev, B., Gnad, F., Macek, B., et al., Global, in vivo, and site-specific phosphorylation dynamics in signaling networks. Cell 2006, 127, 635-648.

[42] Hunter, T., Sefton, B. M., Transforming gene product of Rous sarcoma virus phosphorylates tyrosine. Proc. Natl. Acad. Sci. U S A 1980, 77, 1311-1315.

[43] Villen, J., Beausoleil, S. A., Gerber, S. A., Gygi, S. P., Large-scale phosphorylation analysis of mouse liver. Proc. Natl. Acad. Sci. U S A 2007, 104, 1488-1493.

[44] Gan, C. S., Guo, T., Zhang, H., Lim, S. K., Sze, S. K., A comparative study of electrostatic repulsion-hydrophilic interaction chromatography (ERLIC) versus SCX-IMACbased methods for phosphopeptide isolation/enrichment. J. Proteome Res. 2008, 7, 48694877.

[45] Ouaaz, F., Sola, B., Issaly, F., Kolb, J. P., et al., Growth arrest and terminal differentiation of leukemic myelomonocytic cells induced through ligation of surface CD23 antigen. Blood 1994, 84, 3095-3104.

[46] Oh, I. H., Reddy, E. P., The myb gene family in cell growth, differentiation and apoptosis. Oncogene 1999, 18, 3017-3033.

[47] Lawrence, T., Natoli, G., Transcriptional regulation of macrophage polarization: enabling diversity with identity. Nat. Rev. Immunol. 2011, 11, 750-761.

[48] Eilers, A., Georgellis, D., Klose, B., Schindler, C., et al., Differentiation-regulated serine phosphorylation of STAT1 promotes GAF activation in macrophages. Mol. Cell. Biol. 1995, 15, 3579-3586.

[49] Coccia, E. M., Del Russo, N., Stellacci, E., Testa, U., et al., STAT1 activation during monocyte to macrophage maturation: role of adhesion molecules. Int. Immunol. 1999, 11, 1075-1083.

[50] Jerke, U., Tkachuk, S., Kiyan, J., Stepanova, V., et al., Stat1 nuclear translocation by nucleolin upon monocyte differentiation. PloS one 2009, 4, e8302.

[51] Westin, E. H., Gallo, R. C., Arya, S. K., Eva, A., et al., Differential expression of the amv gene in human hematopoietic cells. Proc. Natl. Acad. Sci. U S A 1982, 79, 2194-2198. 
[52] Gonda, T. J., Metcalf, D., Expression of myb, myc and fos proto-oncogenes during the differentiation of a murine myeloid leukaemia. Nature 1984, 310, 249-251.

[53] Ramsay, R. G., Ikeda, K., Rifkind, R. A., Marks, P. A., Changes in gene expression associated with induced differentiation of erythroleukemia: protooncogenes, globin genes, and cell division. Proc. Natl. Acad. Sci. U S A 1986, 83, 6849-6853.

[54] Clarke, M. F., Kukowska-Latallo, J. F., Westin, E., Smith, M., Prochownik, E. V., Constitutive expression of a c-myb cDNA blocks Friend murine erythroleukemia cell differentiation. Mol.Cell Biol. 1988, 8, 884-892.

[55] Selvakumaran, M., Liebermann, D. A., Hoffman-Liebermann, B., Deregulated c-myb disrupts interleukin-6- or leukemia inhibitory factor-induced myeloid differentiation prior to cmyc: role in leukemogenesis. Mol.Cell Biol. 1992, 12, 2493-2500.

[56] Patel, G., Kreider, B., Rovera, G., Reddy, E. P., v-myb blocks granulocyte colonystimulating factor-induced myeloid cell differentiation but not proliferation. Mol. Cell. Biol. 1993, 13, 2269-2276.

[57] Daigneault, M., Preston, J. A., Marriott, H. M., Whyte, M. K., Dockrell, D. H., The identification of markers of macrophage differentiation in PMA-stimulated THP-1 cells and monocyte-derived macrophages. PloS one 2010, 5, e8668.

[58] Yu, Y., Yoon, S. O., Poulogiannis, G., Yang, Q., et al., Phosphoproteomic analysis identifies Grb10 as an mTORC1 substrate that negatively regulates insulin signaling. Science 2011, 332, 1322-1326.

[59] Burnett, P. E., Barrow, R. K., Cohen, N. A., Snyder, S. H., Sabatini, D. M., RAFT1 phosphorylation of the translational regulators p70 S6 kinase and 4E-BP1. Proc. Natl. Acad. Sci. U S A 1998, 95, 1432-1437.

[60] Nishioka, C., Ikezoe, T., Yang, J., Koeffler, H. P., Yokoyama, A., Blockade of mTOR signaling potentiates the ability of histone deacetylase inhibitor to induce growth arrest and differentiation of acute myelogenous leukemia cells. Leukemia 2008, 22, 2159-2168.

[61] Gery, S., Park, D. J., Vuong, P. T., Virk, R. K., et al., RTP801 is a novel retinoic acidresponsive gene associated with myeloid differentiation. Exp. Hematol. 2007, 35, 572-578. 
[62] Brown, A. L., Salerno, D. G., Sadras, T., Engler, G. A., et al., The GM-CSF receptor utilizes beta-catenin and Tcf4 to specify macrophage lineage differentiation. Differentiation 2012, 83, 47-59.

[63] Tickenbrock, L., Schwable, J., Strey, A., Sargin, B., et al., Wnt signaling regulates transendothelial migration of monocytes. J. Leukoc. Biol. 2006, 79, 1306-1313.

[64] Shibata, T., Chuma, M., Kokubu, A., Sakamoto, M., Hirohashi, S., EBP50, a betacatenin-associating protein, enhances Wnt signaling and is over-expressed in hepatocellular carcinoma. Hepatology 2003, 38, 178-186.

[65] Lilien, J., Balsamo, J., The regulation of cadherin-mediated adhesion by tyrosine phosphorylation/dephosphorylation of beta-catenin. Curr. Opin Cell Biol. 2005, 17, 459-465. [66] Thorne, R. F., Legg, J. W., Isacke, C. M., The role of the CD44 transmembrane and cytoplasmic domains in co-ordinating adhesive and signalling events. J. Cell Sci. 2004, 117, 373-380.

[67] Islas, S., Vega, J., Ponce, L., Gonzalez-Mariscal, L., Nuclear localization of the tight junction protein ZO-2 in epithelial cells. Exp. Cell Res. 2002, 274, 138-148.

[68] Drees, B. E., Andrews, K. M., Beckerle, M. C., Molecular dissection of zyxin function reveals its involvement in cell motility. J. Cell. Biol. 1999, 147, 1549-1560.

[69] Kozasa, T., Jiang, X., Hart, M. J., Sternweis, P. M., et al., p115 RhoGEF, a GTPase activating protein for Galpha12 and Galpha13. Science 1998, 280, 2109-2111.

[70] Gringel, A., Walz, D., Rosenberger, G., Minden, A., et al., PAK4 and alphaPIX determine podosome size and number in macrophages through localized actin regulation. J.Cell. Physiol. 2006, 209, 568-579.

[71] Manabe, R., Kovalenko, M., Webb, D. J., Horwitz, A. R., GIT1 functions in a motile, multi-molecular signaling complex that regulates protrusive activity and cell migration. J. Cell Sci. 2002, 115, 1497-1510.

[72] Ger, M., Zitkus, Z., Valius, M., Adaptor protein Nck1 interacts with p120 Ras GTPaseactivating protein and regulates its activity. Cell. Signal.2011, 23, 1651-1658. 
[73] Rodriguez-Cuenca, S., Carobbio, S., Velagapudi, V. R., Barbarroja, N., et al., Peroxisome proliferator-activated receptor $\mathrm{y}$-dependent regulation of lipolytic nodes and metabolic flexibility. Mol. Cell. Biol. 2012, 32, 1555-1565.

[74] Wang, W., Zhang, X., Zheng, J., Yang, J., High glucose stimulates adipogenic and inhibits osteogenic differentiation in MG-63 cells through cAMP/protein kinase A/extracellular signal-regulated kinase pathway. Mol. Cell. Biochem. 2010, 338, 115-122.

[75] Jia, B., Madsen, L., Petersen, R. K., Techer, N., et al., Activation of protein kinase A and exchange protein directly activated by cAMP promotes adipocyte differentiation of human mesenchymal stem cells. PloS one 2012, 7, e34114.

[76] Ecker, J., Liebisch, G., Englmaier, M., Grandl, M., et al., Induction of fatty acid synthesis is a key requirement for phagocytic differentiation of human monocytes. Proc. Natl. Acad. Sci. U S A 2010, 107, 7817-7822.

[77] Kato, Y., Zhao, M., Morikawa, A., Sugiyama, T., et al., Big mitogen-activated kinase regulates multiple members of the MEF2 protein family. J. Biol Chem. 2000, 275, 1853418540.

[78] Okamoto, S., Li, Z., Ju, C., Scholzke, M. N., et al., Dominant-interfering forms of MEF2 generated by caspase cleavage contribute to NMDA-induced neuronal apoptosis. Proc. Natl. Acad. Sci. U S A 2002, 99, 3974-3979.

[79] Mellor, H., Parker, P. J., The extended protein kinase C superfamily. Biochem. J. 1998, 332 ( Pt 2), 281-292. 
Figure 1. Experimental workflow and analysis. A) Experimental design and workflow. B) The identified phosphopeptides were categorized by their site-localization scores. C) Prevalence of site number per peptide. D) Distribution of phosphosite types.

Figure 1A

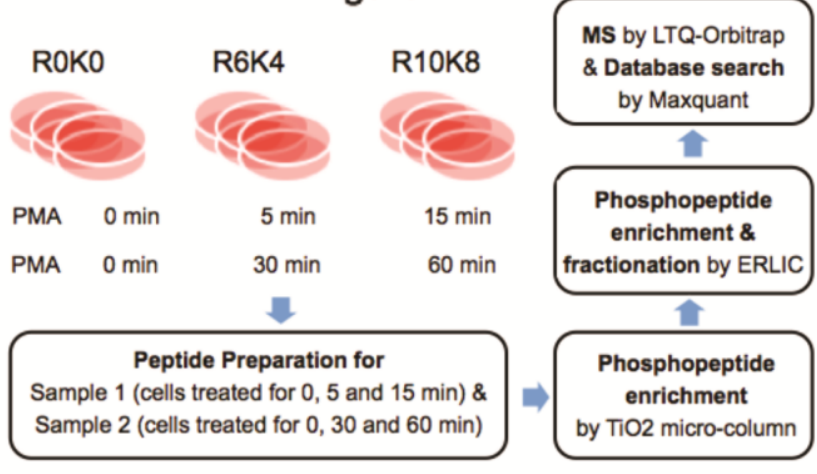

Figure 1C

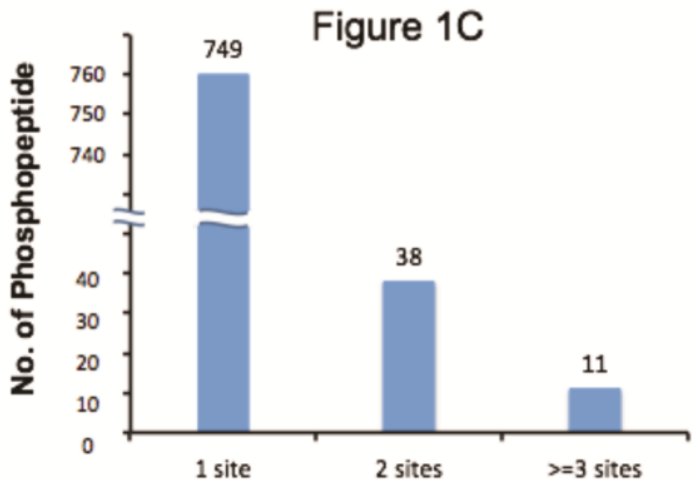

Phosphosite/phosphopeptide

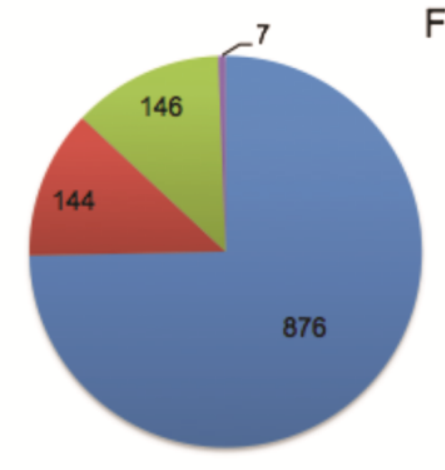

Figure 1B

- Group 1

- Group 2

$=$ Group 3

$=$ Group 4

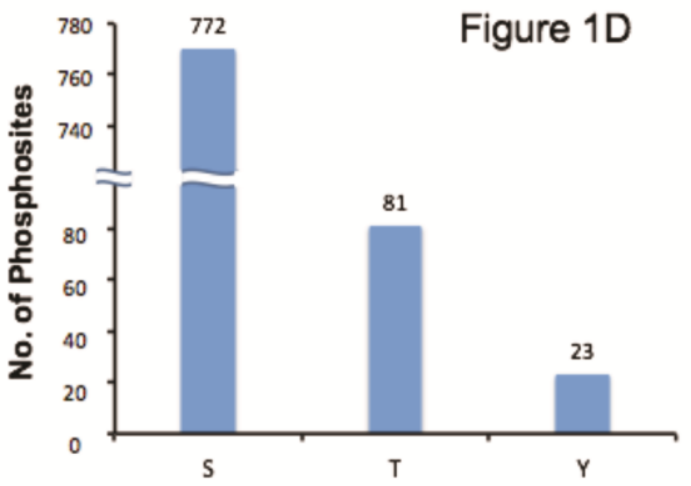

Phosphosite type 
Figure 2. SWATH-MS analysis verified the expression patterns of 24 phosphopeptides quantified by SILAC experiments. The SILAC ratio (left y-axis) is indicated using blue square (घ) and the SWATH quantitation by peak area of the peptide product ions (right y-axis) is indicated by the red triangle ( ) and presented by mean +/- S.D. from three technical replicates. The $\mathrm{x}$-axis indicates the PMA-treatment time for five different time points.

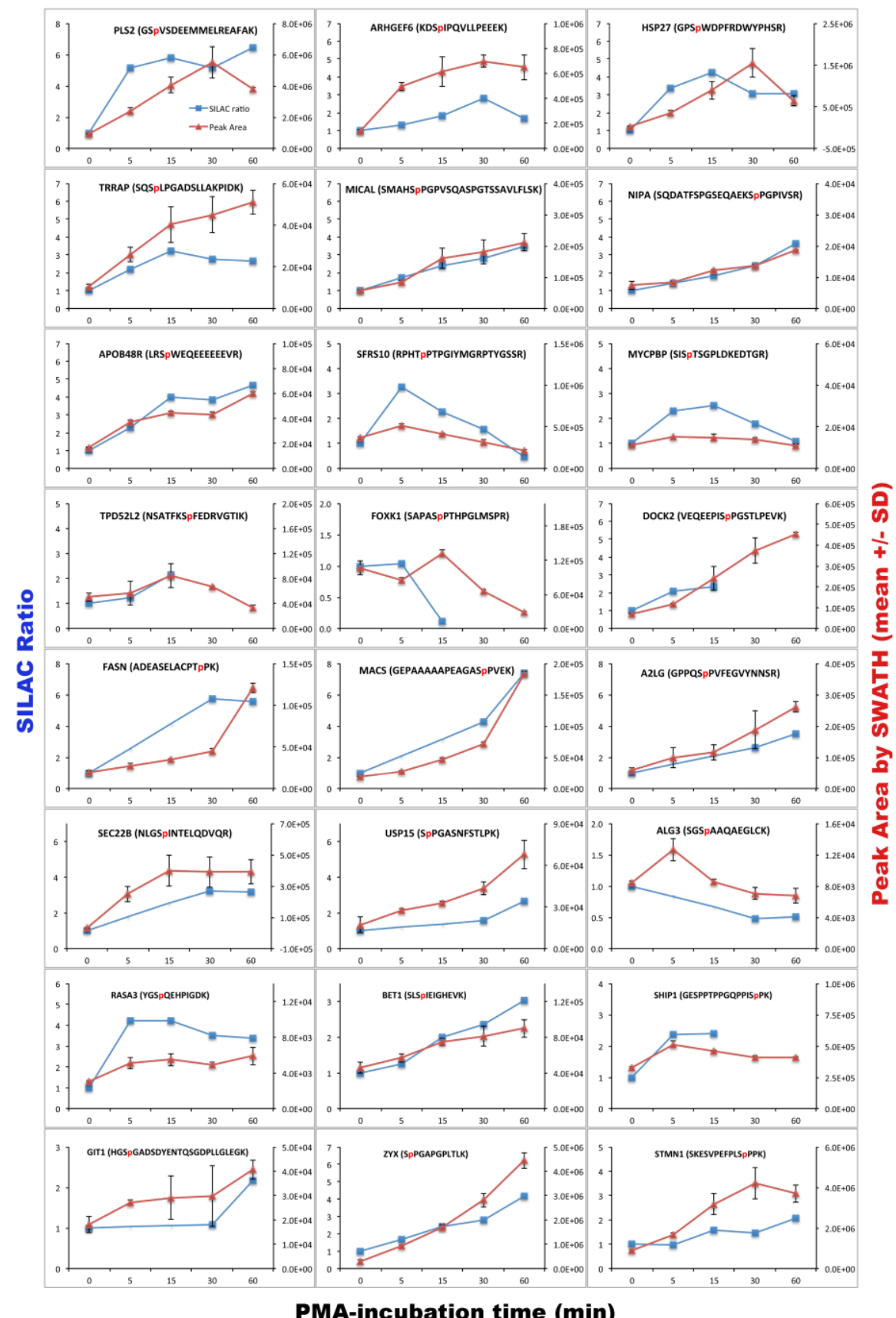


Figure 3. Gene Ontology (GO) classification of the identified PMA-regulated and nonregulated phosphoproteins into the cellular component $(A)$, biological process $(B)$ and molecular function $(C)$ group. Left and right panels are $\mathrm{GO}$ classifications for regulated phosphoproteins and non-regulated phosphoproteins respectively.
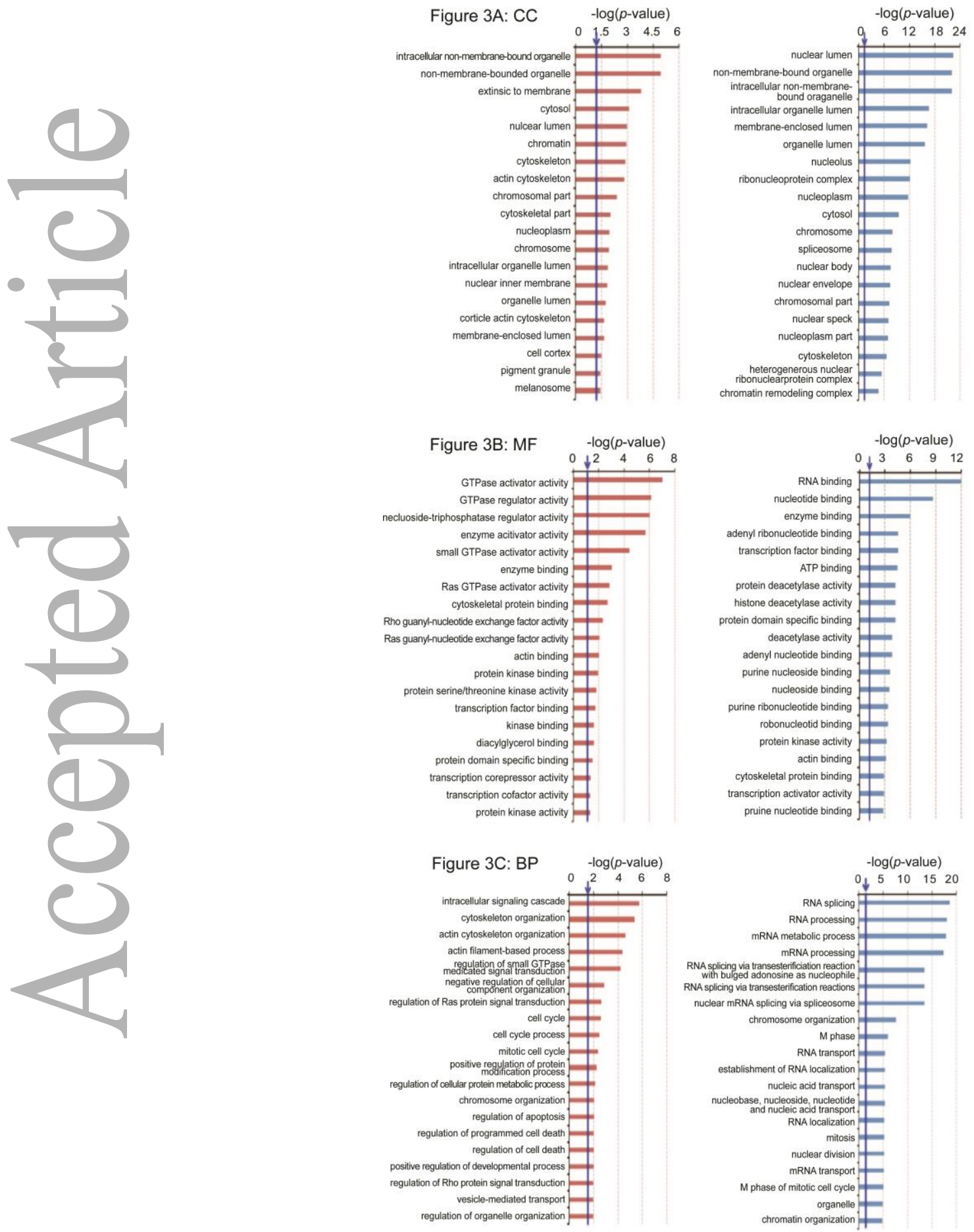
Figure 4. SWATH-MS analysis identified increased phosphorylation at the Ser727 residue of STAT1 and decreased phosphorylation at the Ser1163 residue of MYBBP1A during the early differentiation stages.
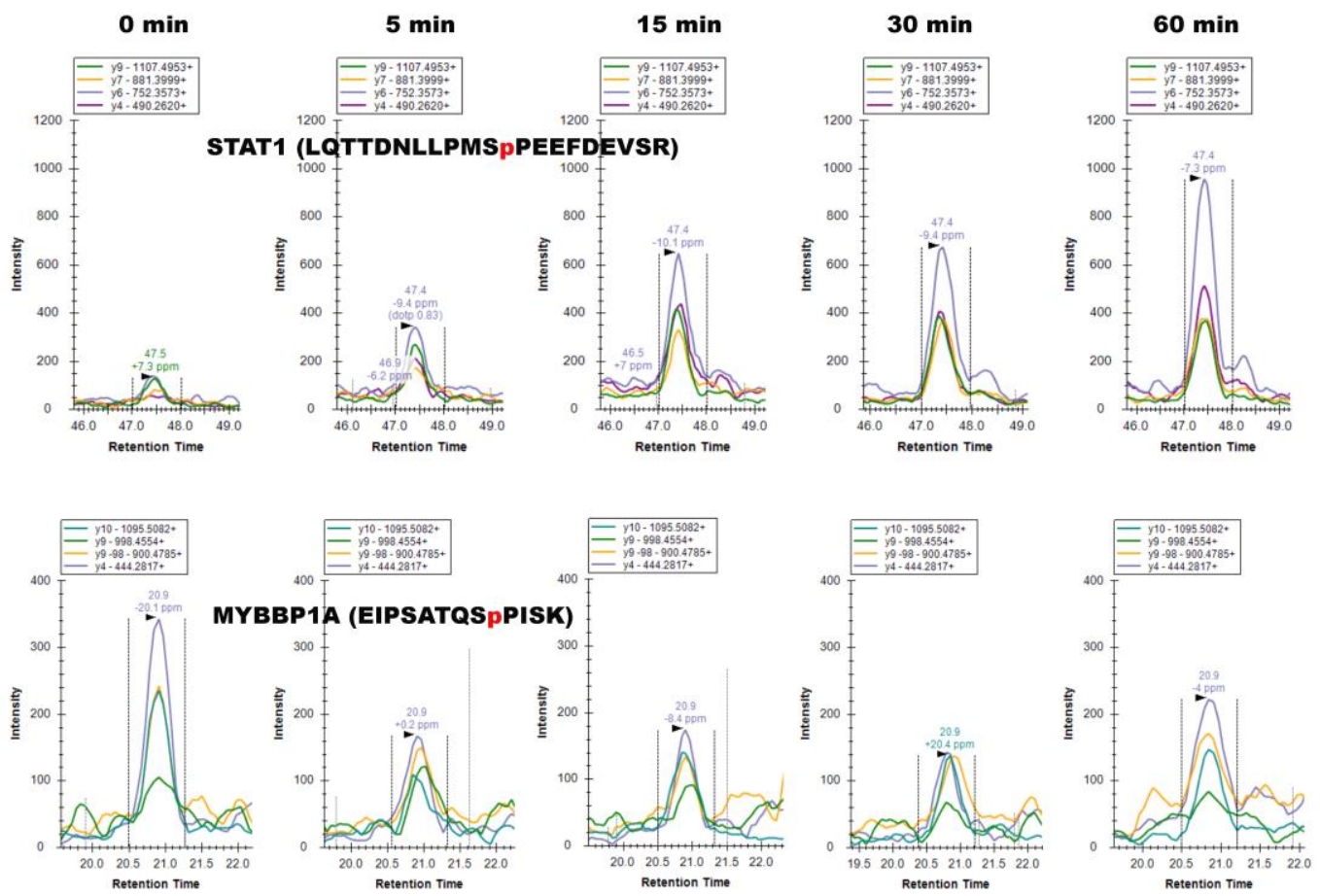
Figure 5. Protein-protein interaction network of regulated phosphoproteins. The five functional clusters are shown in different colors and their central regulators (hubs) are positioned at the center of each sub-network. Each node size is proportional to the node degree.

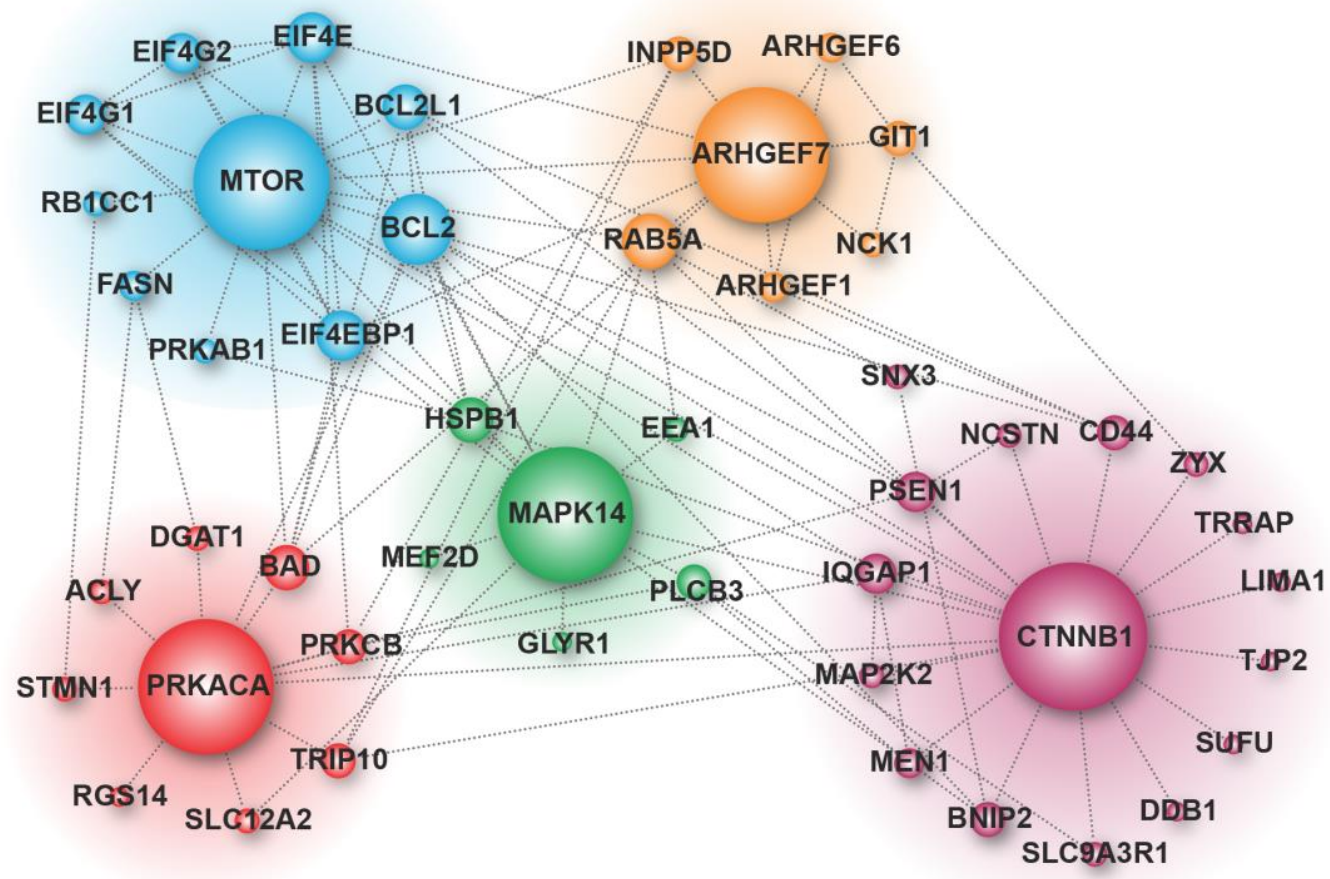

